\title{
Measurement of diffractive and exclusive processes with the ATLAS detector
}

\author{
Grzegorz Gach ${ }^{1, a}$ on behalf of the ATLAS Collaboration. \\ ${ }^{1}$ AGH University of Science and Technology
}

\begin{abstract}
The ATLAS Collaboration has carried out a study of diffractive dijet production in proton-proton collisions at a centre-of-mass energy of $\sqrt{s}=7 \mathrm{TeV}$ at the LHC. The data distributions are compared with Monte Carlo models and the rapidity gap survival probability has been estimated in the kinematic region with high diffractive contribution. Prospects for exclusive jet production studies with the forward proton tagging capability of the AFP sub-detector of ATLAS are also discussed. First results based on data taken jointly with the ATLAS and the LHCf detectors in a $\mathrm{p}+\mathrm{Pb}$ run will also be shown. In addition, the measurement of the cross-section for the exclusive production of di-lepton pairs in $p p$ collisions at $\sqrt{s}=7 \mathrm{TeV}$ is discussed.
\end{abstract}

\section{Introduction}

Diffractive interaction can be defined as a process in which an object carrying vacuum quantum numbers is exchanged. In case of proton-proton collisions such interaction can be divided into three main classes:

- single diffractive dissociation - when one of the incoming protons emerges from the interaction and the other one dissociates into a system $X$ which quantum numbers are the same as of incoming proton,

- double diffractive dissociation - when each of the incoming protons dissociate into systems $X$ and $Y$, respectively, each of the systems has proton quantum numbers,

- central production - when a system $Z$ is produced in the final state and the incoming protons can dissociate into separate hadronic systems with proton quantum numbers or stay intact.

In the non-diffractive interactions the produced systems and proton remnants are not clearly separated, because the mediating particle carries a charge and thus radiates particles and fills the space between the systems. This does not happen in the diffractive interactions and thus the probability of the dissociated systems to be well separated in pseudorapidity is relatively large. In cases when it is not possible to measure the scattered proton, this property is utilised to experimentally identify the diffractive interactions.

ae-mail: grzegorz.gach@cern.ch 


\section{Dijet Production with Large Rapidity Gaps at $7 \mathrm{TeV}$}

The ATLAS [1] Collaboration published first studies of the single diffractive dissociation at $\sqrt{s}=7$ $\mathrm{TeV}$ already in 2012 [2]. It was an inclusive measurement without the requirement of a hard scale. Since there is no information about the scattered proton, a rapidity gap i.e. a region in $\eta^{1}$ devoid of any hadronic activity, was required between the edge of the calorimeter (which covers $|\eta|<4.9$ ) and the nearest energy deposit which is not identified as noise. This procedure provides two rapidity gaps: one in the forward and one in the backward direction. In these studies the larger one is used as the rapidity gap, because single diffractive dissociation events are studied and background contributions are subtracted.

The measurement of dijet production in events with large rapidity gaps [3] is an extension of the study performed in ref. [2]. The presence of jets provides a hard scale in the interaction and allows comparisons with perturbative calculations. This process gives an insight into the underlying parton dynamics and colour singlet exchange. It is also sensitive to the soft survival probability $S^{2}$ [4].

The measurement is based on the low pile-up data with $\langle\mu\rangle \sim 0.04-0.14$. The jets were reconstructed using the anti- $k_{t}$ algorithm with two radii: $R=0.6$ and $R=0.4$. The latter results are not presented in the paper but are available at HepData [5]. Each jet was required to have transverse momentum greater than $20 \mathrm{GeV}$ and pseudorapidity between -4.4 and 4.4.

The measured differential cross-section for dijet production as a function of rapidity gap $\left(\Delta \eta^{F}\right)$ is presented in Fig. 1. In contrast to the soft rapidity gap result no clear diffractive plateau can be observed. This is caused by the reduction of phase space for the dijet system to develop with a growing rapidity gap. The measured cross-section is compared with Pythia 8 Monte Carlo with the ATLAS tune AU2-CT10 [6]. The non-diffractive contribution was scaled by $1 / 1.4$ so that the predicted cross-section for dijet production with a rapidity gap between 0 and 0.5 is the same as the measured one. This region was chosen, because most of the events with a small rapidity gap are expected to have a non-diffractive origin. The Monte Carlo describes the data well in the whole measured rapidity gap range. According to the Pythia 8 predictions the diffractive and non-diffractive cross-sections are equal for rapidity gaps close to 3 . Larger rapidity gaps are dominated by the contribution from single diffractive dissociation.

Diffractive and non-diffractive distributions can also be separated based on a variable

$$
\tilde{\xi} \simeq M_{X}^{2} / s=\sum_{\text {particles }}\left(p_{T} e^{-|\eta|}\right) / \sqrt{s}
$$

where $M_{X}$ is the mass of the proton dissociated system, $p_{T}$ is the particle transverse momentum and $\eta$ is the particle pseudorapidity. The sum runs over all particles in the hadronic final state. In Fig. 1 the distribution of $\log _{10}(\tilde{\xi})$ is presented and it can be observed that the smaller the logarithm the larger the diffractive contribution. Both of the methods introduced above to improve purity of diffractive sample were used in further studies i.e. rapidity gap $\Delta \eta^{F}>2$ and $\log _{10}(\tilde{\xi})<-2$ were required.

The comparison of POMWIG [7] prediction with the data is presented in Fig. 2. The predicted cross-section is about three times larger than the measured one. This discrepancy can be fixed by modifying the rapidity gap survival probability, which in the basic POMWIG is equal to one. The extracted probability is model dependent and was measured in the region of $-3.2<\log _{10}(\tilde{\xi})<-2.5$, where the contribution from single diffractive dissociation is expected to dominate by far. The proba-

\footnotetext{
${ }^{1}$ ATLAS uses a right-handed coordinate system with its origin at the nominal interaction point (IP) in the centre of the detector and the z-axis along the beam pipe. The x-axis points from the IP to the cetnre of the LHC ring, and the y-axis points upward. Cylindrical coordinates $(r, \phi)$ are used in the transverse plane, $\phi$ being the azimuthal angle around the beam pipe. The pseudorapidity is defined in terms of the polar angle $\theta$ as $\eta=-\ln \tan (\theta / 2)$.
} 

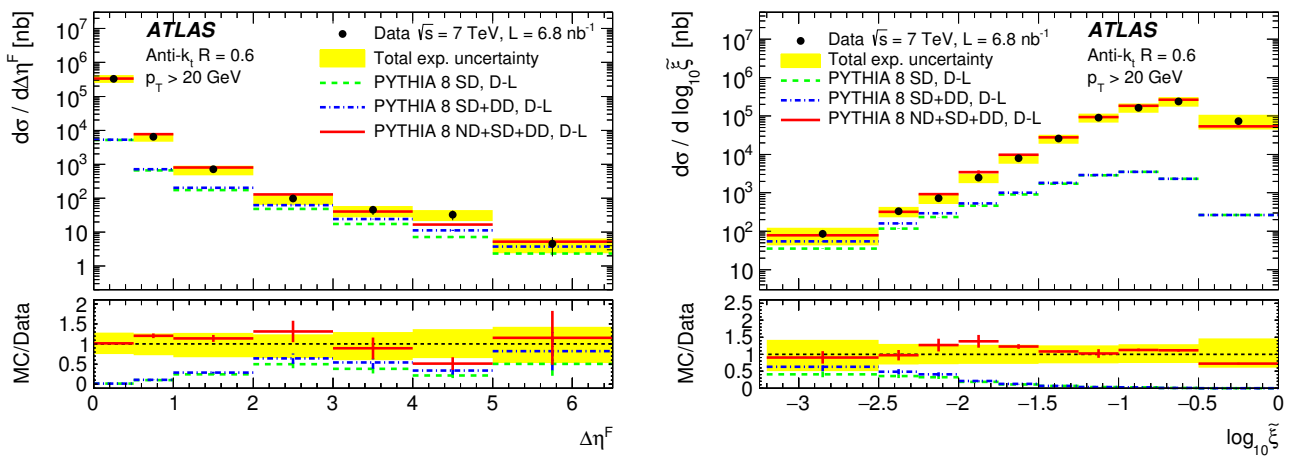

Figure 1. Differential cross-sections for dijet production [3] as functions of rapidity gap (left) and $\log _{10}(\tilde{\xi})($ right), where $\tilde{\xi} \simeq M_{X}^{2} / s$. The rapidity gap is defined as a distance between the calorimeter edge and the nearest energy deposit not identified as noise.
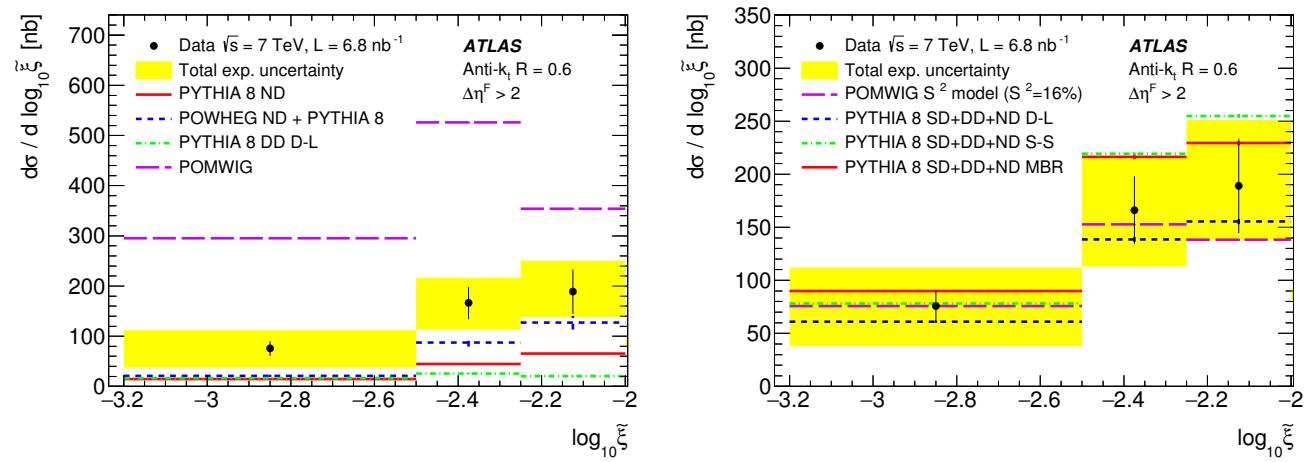

Figure 2. Differential cross-sections for dijet production [3] with rapidity gap greater than 2 as a function of $\log _{10}(\tilde{\xi})$, where $\tilde{\xi} \simeq M_{X}^{2} / s$. The left plot shows comparison between the measured cross-sections and POMWIG MC that was used to extract rapidity gap survival probability. The right plot shows the comparison between the data and predictions obtained with three Pomeron flux models.

bility was measured to be $S^{2}=0.16 \pm 0.4$ (stat.) \pm 0.8 (syst.). It is worth noting that Pythia $8 \mathrm{MC}$ does not need any additional rapidity gap survival probability in order to describe the data well.

The measured dijet production differential cross-section as a function of $\log _{10}(\tilde{\xi})$ was used to test Pomeron flux models. Pythia $8 \mathrm{MC}$ with: Donnachie and Landshoff (D-L) [8], Schuler and Sjöstrand (S-S) [9] and Minimum Bias Rockefeller (MBR) [10] is compared with the data in Fig. 2. All predictions agree with the measurements within the experimental uncertainties.

\section{Exclusive Dijet Production}

One of the possible follow-ups of the dijet production with a large rapidity gap is the measurement of exclusive dijet production. In this process both of the incoming protons stay intact and a gluon pair, which hadronises into a dijet system, is produced. There are no particles that would not be part 

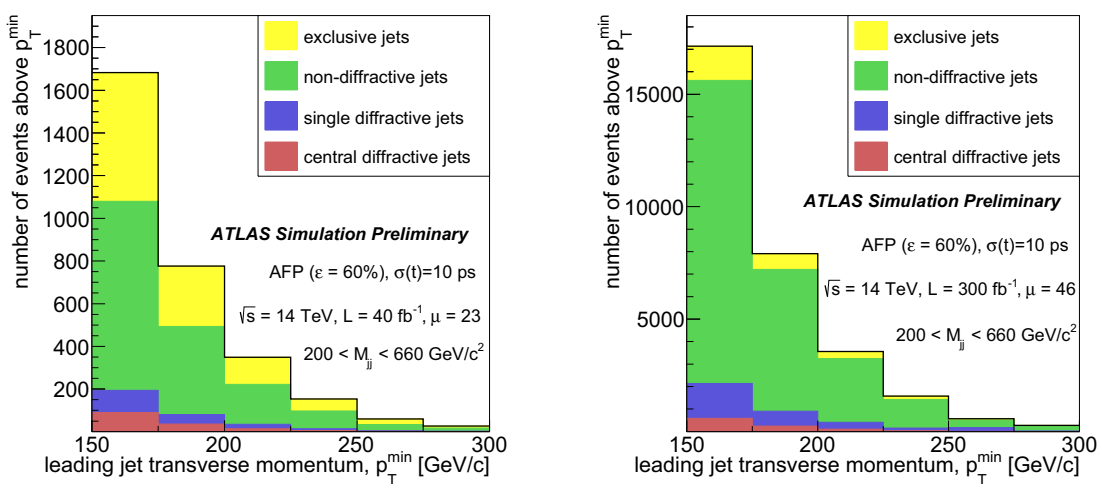

Figure 3. Results of exclusive dijet production feasibility studies [11]. The number of events, separated into signal and background categories, as a function of the leading jet transverse momentum is presented. The left plot shows studies with medium pile-up conditions of $\langle\mu\rangle=23$. The right plot presents results for high pile-up conditions $\langle\mu\rangle=46$.

of the dijet system. Only gluons take part in this process, thus this measurement provides a way to probe their distributions in proton. The measurement of exclusive dijet production is very challenging, because of relatively low cross-section $(\sigma=0.5 \mathrm{pb})$ and large backgrounds [11]. The scattered proton tagging is a must in this measurement. In the future it will be provided by the ATLAS Forward Proton (AFP) detectors [12], which were approved by the Research Board in 2015 and will be installed 220 $\mathrm{m}$ away from the nominal interaction point. The AFP stations will be equipped with timing detectors which are crucial for measurements in high pile-up conditions.

The feasibility studies of this measurement are published in an ATLAS note [11]. They reveal that the main source of uncertainty is the background estimation. It was assessed that in case of medium pile-up conditions $(\langle\mu\rangle=23)$ and measurement of both scattered protons the signal to background ratio is 0.57 . The number of events, separated into signal and background categories, as a function of the leading jet transverse momentum is presented in the left plot in Fig. 3. This proves that although the measurement will be difficult, it is nonetheless feasible. The same studies were repeated for high pile-up conditions of $\langle\mu\rangle=46$. The results are presented in the right plot in Fig. 3. The estimated signal to background ratio is 0.16 , which makes the measurement virtually impossible with this technique.

\section{Exclusive Dilepton Production}

In the previous section it was shown that measurements of exclusive processes are very challenging. Yet, the ATLAS Collaboration successfully measured the two-photon exclusive production of lepton pairs [13]. The studies were done using proton-proton collisions at $\sqrt{s}=7 \mathrm{TeV}$ and mean pile-up between 6.3 and 11.6. The luminosity of the data amounts to $4.6 \mathrm{fb}^{-1}$. A clean sample of signal events was selected by requiring exactly two opposite charge tracks with transverse momentum greater than $400 \mathrm{MeV}$. The net transverse momentum of the tracks pair had to be smaller than $1.5 \mathrm{GeV}$ and the centre-of-mass energy of the system could not be in the Z-boson mass region i.e. $m_{l l} / \mathrm{GeV} \notin(70,105)$.

The measurement was performed in electron and muon channels. Electrons with transverse momentum greater than $12 \mathrm{GeV}$ and pseudorapidity within the range of -2.4 and 2.4 were used in this analysis. The exclusive electron-positron production cross-section was measured to be 


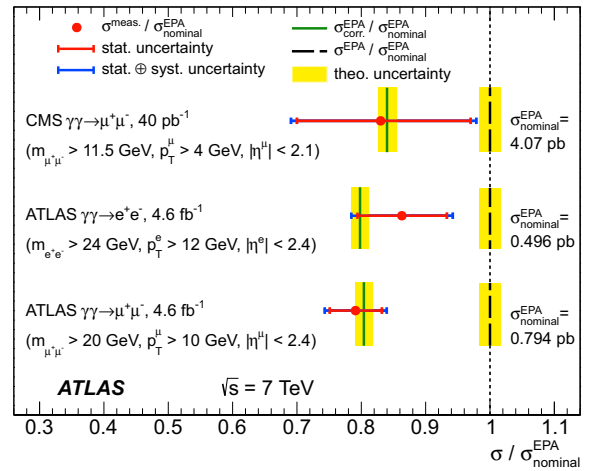

Figure 4. A summary of exclusive dilepton production measurements done by the ATLAS and CMS collaborations [13]. The measured and predicted cross-sections are normalised to the results of calculations based on the uncorrected Equivalent Photon Approximation model.

$\sigma_{\gamma \gamma \rightarrow e^{+} e^{-}}=0.428 \pm 0.035$ (stat.) \pm 0.018 (syst.) pb. This result is in agreement with the prediction of the Equivalent Photon Approximation (EPA) model corrected for the proton absorption effects $\sigma_{\gamma \gamma \rightarrow e^{+} e^{-}}^{\mathrm{EPA}}=0.398 \pm 0.005$ (theor.) pb.

The muon channel measurement was based on the particles identified as muons and with transverse momentum greater than $10 \mathrm{GeV}$. The tracks had to be reconstructed with $|\eta|<2.4$. The measured cross-section $\sigma_{\gamma \gamma \rightarrow \mu^{+} \mu^{-}}=0.628 \pm 0.032$ (stat.) \pm 0.021 (syst.) pb agrees with the (EPA) model corrected for the proton absorption effects $\sigma_{\gamma \gamma \rightarrow \mu^{+} \mu^{-}}^{\mathrm{EPA}}=0.638 \pm 0.011$ (theor.) pb.

In Fig. 4 a summary of exclusive dilepton production measurements is presented. The CMS measurement in the muon channel suggests that absorption corrections may play an important role in this process, but it is in agreement with uncorrected EPA prediction within a little bit more than one standard deviation. The ATLAS measurement in the same channel proves that the Equivalent Photon Approximation model alone is not sufficient to describe the data and the absorption corrections are crucial. The measured suppression with respect to the uncorrected EPA is of the order of $20 \%$.

\section{First Results from ATLAS and LHCf Experiments Combination}

The LHCf experiment [14] consists of detectors placed about 140 metres away from the nominal interaction point that measure only neutral particles with pseudorapidity smaller than -8.4 . Its main purpose was to measure particle showers. The first successful attempt of combining the data from the ATLAS and LHCf detectors is published in an ATLAS note [15].

The data from proton-lead runs at centre-of-mass energy of $5.02 \mathrm{TeV}$ were used. Approximately 127000 common events were recorded. Thanks to the combination of information from both detectors it was possible to measure the energy spectra of neutral particles produced at very small angles with and without activity in the central rapidity region. These results are presented in the left plot in Fig. 5. Additionally, it was possible to measure the scattering angle of these particles (the right plot in Fig. 5). In the majority of measured events no activity in the central rapidity region is observed which suggests ultra-peripheral-collisions. In such events neutral hadron-like particles energy distribution peaks at 3.5 $\mathrm{TeV}$ and scattering angle of these particles is close to zero. This indicates that the dominating process is the production of the $\Delta^{+}$baryon: $p+\gamma \rightarrow \Delta^{+} \rightarrow n+\pi^{+}$. 

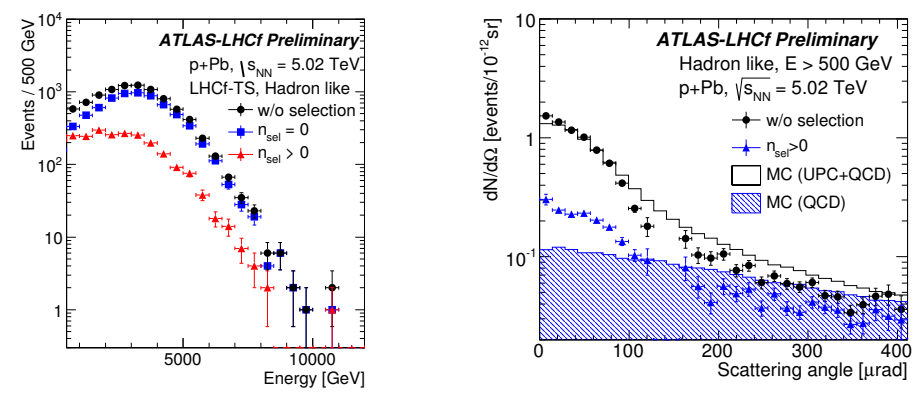

Figure 5. Results of the first ATLAS and LHCf data combination [15]. The left plot shows the energy spectrum of hadron-like neutral particles with and without activity in the central rapidity region. The right plot presents the scattering angle of these particles.

\section{Summary}

ATLAS diffractive and exclusive physics program is rich and consistent. The first diffractive measurement of soft rapidity gaps was followed by an analysis of the dijet production with a large rapidity gap. It turned out that a tuned Pythia 8 is able to describe the data. The diffractive dijet measurement is planned to be extended by the exclusive dijet production studies with AFP detectors. From the published exclusive measurement of dilepton production it can be concluded that absorption corrections are crucial. The first combined measurement with the ATLAS and the LHCf detectors has been published.

\section{Acknowledgements}

This work was supported in part by the National Science Center grants DEC-2011/03/B/ST/02631.

\section{References}

[1] ATLAS Collaboration, JINST 3, S08003 (2008)

[2] ATLAS Collaboration, Eur. Phys. J. C72, 1926 (2012), 1201.2808 [hep-ex]

[3] ATLAS Collaboration, CERN-PH-EP-2015-227 (2015), 1511.00502 [hep-ex]

[4] J.D. Bjorken, Phys. Rev. D 47, 101 (1993)

[5] The Durham HepData Project, http://hepdata.cedar.ac.uk/

[6] ATLAS Collaboration, ATL-PHYS-PUB-2012-003 (2012), https://cds.cern.ch/record/1474107

[7] B.E. Cox, J.R. Forshaw, Comput. Phys. Commun. 144, 104 (2002), hep-ph/0010303

[8] A. Donnachie, P. Landshoff, Nuclear Physics B 244, 322 (1984)

[9] G.A. Schuler, T. Sjöstrand, Phys. Rev. D 49, 2257 (1994)

[10] R. Ciesielski, K. Goulianos, PoS ICHEP2012, 301 (2013), 1205. 1446 [hep-ex]

[11] ATLAS Collaboration, ATL-PHYS-PUB-2015-003 (2015), https://cds.cern.ch/record/1993686

[12] L. Adamczyk et al., Tech. Rep. ATL-COM-LUM-2011-006, CERN, Geneva (2011), AFP technical proposal, https://cds. cern.ch/record/1331213

[13] ATLAS Collaboration, Phys. Lett. B749, 242 (2015), 1506.07098 [hep-ex]

[14] O. Adriani et al. (LHCf), JINST 3, S08006 (2008)

[15] ATLAS Collaboration, ATL-PHYS-PUB-2015-038 (2015), https://cds.cern.ch/record/2047832 